\title{
Pioglitazone inhibits the expression of matrix metalloproteinase-9, a protein involved in diabetes-associated wound healing
}

\author{
JUN ZHANG $^{1}$, XIAOYUAN HUANG ${ }^{1}$ and LINGFENG WANG ${ }^{2}$ \\ ${ }^{1}$ Department of Burns and Plastic Surgery, Changsha Xiangya Hospital, Central South University, Changsha, Hunan 410008; \\ ${ }^{2}$ Department of Burn, Inner Mongolia Institute of Burn, Baotou Steel Hospital, Baotou, Inner Mongolia 014010, P.R. China
}

Received August 21, 2013; Accepted March 31, 2014

DOI: $10.3892 / \mathrm{mmr} .2014 .2277$

\begin{abstract}
Matrix metalloproteinase-9 (MMP-9) is a protein involved in diabetes-associated wound healing. The present study aimed to determine whether pioglitazone, an agonist of peroxisome proliferator-activated receptor- $\gamma$ (PPAR- $\gamma$ ), inhibits the expression of MMP-9. HaCaT cells at a density of $6 \times 10^{5}$ cells/well were seeded into 6 -well plates in medium and were cultured for $24 \mathrm{~h}$. The cells were then treated with bovine serum albumin (BSA) only or advanced glycation end-product (AGE)-BSA $(50,100,200,300$ or $400 \mu \mathrm{g} / \mathrm{ml})$, with or without pioglitazone $(0.5$ or $1 \mu \mathrm{M})$. The effects of AGE-BSA on cell viability were determined by the 3-(4,5-dimethylthiazol-2-yl)2,5-diphenyltetrazolium bromide assay. The levels of MMP-9 secreted into the medium were detected by an enzyme-linked immunosorbent assay. The mRNA and protein levels were analyzed by quantitative polymerase chain reaction (qPCR) and western blot analysis, respectively. AGEs are able to increase the level of MMP-9 mRNA in HaCaT cells and the levels of MMP-9 protein secreted into the medium. Pioglitazone $(0.5$ or $1 \mu \mathrm{M}$ ) significantly inhibited the levels of MMP-9 in the treated HaCaT cells. Pioglitazone $(0.5$ or $1 \mu \mathrm{M})$ also suppressed the levels of MMP-9 in the cell culture medium. Pioglitazone at concentrations of 0.5 and $1 \mu \mathrm{M}$ significantly suppressed the levels of MMP-9 mRNA to 20 or $8 \%$, respectively. These results suggest that pioglitazone is able to effectively suppress the expression of MMP-9 via a transcriptional mechanism.
\end{abstract}

\section{Introduction}

Impaired wound healing is frequently reported in patients with diabetes. The increase in matrix metalloproteinase-9 (MMP-9) expression is associated with diabetes-associated

Correspondence to: Professor Xiaoyuan Huang, Department of Burns and Plastic Surgery, Changsha Xiangya Hospital, Central South University, 87 Xiangya Road, Changsha, Hunan 410008, P.R. China

E-mail: zhjun2685@sohu.com

Key words: pioglitazone, matrix metalloproteinase-9, diabetes, wound healing wound healing (1). Previous experimental results indicate that MMP-9 expression is elevated during wounding in diabetic rats (2). Furthermore, exogenous MMP-9 expression is able to exacerbate chronic wounding (3). Since increased levels of MMP-9 are a factor contributing to poor wound healing in diabetic foot ulcers $(4,5)$, reagents that decrease MMP-9 expression levels may be a useful method to cure the impaired wound healing in diabetic patients.

Pioglitazone is an agonist of the peroxisome proliferator-activated receptor- $\gamma$ (PPAR- $\gamma$ ) that is a ligand-dependent transcription factor (6). The activation of PPAR- $\gamma$ protects pancreatic $\beta$-cells from cytotoxicity by preventing nuclear factor (NF) $-\kappa B$ activation (7-10). Pioglitazone is an antidiabetic agent, which improves insulin production in patients with diabetes. Pioglitazone also increases insulin sensitivity, thus it elevates glucose uptake and inhibits hepatic glucose output (11). It has been reported that pioglitazone is also able to reduce oxidative stress (12-16).

Advanced glycation end-products (AGEs) are a group of heterogeneous compounds that are derived from the non-enzymatic reaction of reducing sugars with proteins, lipids or nucleic acids (17). AGEs contribute to the development of various vascular diabetic complications through increasing the production of reactive oxygen species (ROS), the formation of cross-links between molecules in the basement membrane and the extracelluar matrix, and by affecting various cellular signaling pathways through the receptor for AGEs (RAGE) (18). The binding of AGEs to RAGE triggers oxidative stress and activates the transcription factor $\mathrm{NF}-\kappa \mathrm{B}$, thus, promoting the expression of pro-inflammatory mediators and local cellular responses $(19,20)$.

In the present study, human keratinocytes were treated with pioglitazone in the presence of AGEs. It was demonstrated that pioglitazone decreases the expression of MMP-9 induced by the treatment of AGEs. The results suggest that pioglitazone may have therapeutic effects on impaired wound healing associated with diabetes via a mechanism of inhibiting the expression level of MMP-9.

\section{Materials and methods}

Cells and reagents. Human HaCaT keratinocytes, which were provided by Xiangya Hospital (Changsha, China), were cultured in Dulbecco's modified Eagle's medium (DMEM) 
supplemented with $10 \%$ fetal bovine serum (Gibco-BRL, Carlsbad, CA, USA), penicillin (100 U/ml) and streptomycin $(100 \mu \mathrm{g} / \mathrm{ml})$. For the serum-starving experiments, cells at $80 \%$ confluence were cultured by overnight incubation in serum-free DMEM containing $0.5 \mathrm{mg} / \mathrm{ml}$ bovine serum albumin (BSA; Calbiochem, La Jolla, CA, USA). Pioglitazone and AGE-BSA were purchased from Sigma (St. Louis, MO, USA). Antibodies against MMP-9 and $\beta$-actin were purchased from Santa Cruz Biotechnology, Inc. (Santa Cruz, CA, USA).

Cell treatments and the 3-(4,5-dimethylthiazol-2-yl)2,5-diphenyltetrazolium bromide (MTT) assay. HaCaT cells at a density of $6 \times 10^{5}$ cells/well were seeded into 6 -well plates in medium and were cultured for $24 \mathrm{~h}$. The cells were then treated with BSA only or AGE-BSA (50, 100, 200, 300 and $400 \mu \mathrm{g} / \mathrm{ml})$, with or without pioglitazone $(0.5 \mu \mathrm{M})$. At the end of each experiment, cells were incubated with $0.5 \mathrm{mg} / \mathrm{ml}$ MTT at $37^{\circ} \mathrm{C}$ for $4 \mathrm{~h}$. The MTT kit was purchased from Invitrogen Life Technologies (Carlsbad, CA, USA). The supernatants were discarded and $50 \mu 1$ dimethylsulfoxide was added into each well. The 96-well plates (Asahi Glass Corp, Tokyo, Japan). were agitated for $10 \mathrm{~min}$. The growth status and morphological changes of the cells were detected under an inverted microscope (Olympus, Tokyo, Japan). The absorbance was determined at $540 \mathrm{~nm}$ using a Synergy HT microplate reader (Molecular Devices, Sunnyvale, CA, USA). The viability of treated cells was expressed relative to the control cells treated with BSA (relative viability).

Quantitative polymerase chain reaction ( $q P C R)$. Total RNA was harvested from cells using the RNeasy mini kit (Qiagen, Valencia, CA, USA) according to the manufacturer's instructions. RNA $(1 \mu \mathrm{l})$ was reverse transcribed into cDNA using random primers with a Reverse Transcription II system purchased from Promega Corporation (Madison, WI, USA) according to the manufacturer's instructions. qPCR was conducted using an ABI Prism Sequence Detection system (Applied Biosystems, Foster City, CA, USA). An assay reagent containing premixed primers and a VIC-labeled probe (Applied Biosystems; cat. no. 4310884E) was used to quantify the expression of endogenous GAPDH mRNA. The amplification of the MMP-9 cDNA and the endogenous GAPDH cDNA was determined with FAM and VIC fluorescent intensities, respectively. The relative quantity of MMP-9 transcripts was normalized to the quantity of GAPDH mRNA at the same conditions. The primers used were as follows: Forward: 5'-GCACGACGTCTTCCAGTACC-3' and reverse: 5'-CAGGATGTCATAGGTCACGTAGC-3' for MMP-9. The experiments were repeated independently at least three times.

Immunoblotting assays. Total proteins were harvested from supernatants or from cells, separated on 10\% SDS-PAGE gels and then subjected to immunoblot analysis. The primary antibodies against MMP-9 and $\beta$-actin were purchased from Santa Cruz Biotechnology, Inc. (anti-MMP-9; cat. no. sc-21733; 1:200; anti- $\beta$-actin; cat. no. sc-130301; $1: 10,000)$. The secondary antibodies used in the present study were goat anti-mouse IgG conjugated to horseradish peroxidase antibodies (cat. no. sc-2005; 1:10,000; Santa Cruz Biotechnology, Inc.). Bound antibodies were detected using an enhanced chemiluminescence system (Pierce Biotechnology, Inc., Rockford, IL, USA). The experiments were repeated independently at least three times. Image quantifications were performed using ImageQuant software (GE Healthcare Life Sciences, Piscataway, NJ, USA).

Enzyme-linked immunosorbent assay (ELISA). The MMP-9 concentrations in the medium of the HaCaT keratinocytes treated with BSA, AGE-BSA or in the presence or absence of pioglitazone $(0.5$ or $1 \mu \mathrm{M})$ were determined using commercially available ELISA kits (MMP-9 ELISA kit; Raybiotech, Norcross, GA, USA). The experiment was repeated independently at least six times. The values are expressed as the mean \pm standard deviation (SD).

Statistical analysis. The experimental data are expressed as the mean \pm SD. Statistical software (SPSS 10.0; SPSS, Inc., Chicago, IL, USA) was used for independent sample t-tests. $\mathrm{P}<0.05$ was considered to indicate a statistically significant difference.

\section{Results}

AGEs increase the expression of MMP-9 transcripts in HaCaT cells. AGEs contribute to the development of various vascular diabetic complications and high MMP-9 expression exacerbates chronic wounds. To determine whether AGE-BSA affects the expression of MMP-9 in HaCaT cells, the HaCat cells were treated with BSA only or AGE-BSA (50, 100, 200, 300 and $400 \mu \mathrm{g} / \mathrm{ml}$ ) for $24 \mathrm{~h}$. As shown in Fig. 1A, AGE-BSA treatment was not observed to significantly affect the cell viability, indicating that such dosages of AGE-BSA do not result in non-specific cytotoxicity. However, the qPCR results (Fig. 1B) indicated that AGE-BSA at concentrations of 300 or $400 \mu \mathrm{g} / \mathrm{ml}$ markedly increased the transcript levels of MMP-9 in the cells. These results suggest that AGEs are able to increase the level of MMP-9 mRNA in HaCaT cells.

AGEs increase the levels of MMP-9 protein secreted into the medium. To determine whether AGEs increase the levels of MMP-9 protein secreted into the medium, $\mathrm{HaCaT}$ cells were seeded into 6 -well plates at a density of $6 \times 10^{5}$ cells/well, serum-starved overnight and then cultured for $24 \mathrm{~h}$ with various concentrations (50, 100, 200, 300 and $400 \mu \mathrm{g} / \mathrm{ml})$ of AGE-BSA or unmodified BSA. The medium was collected $24 \mathrm{~h}$ after treatment with AGE-BSA or unmodified BSA. The levels of MMP-9 in the culture were detected by ELISA. As shown in Fig. 2A, the ELISA results suggested that AGE-BSA increased the levels of MMP-9 in the medium. The levels of MMP-9 secreted into the medium were upregulated by up to four-fold upon treatment with AGE-BSA at a concentration of $400 \mu \mathrm{g} / \mathrm{ml}$. The western blot analysis results (Fig. 2B) also indicated that AGE-BSA (300 or $400 \mu \mathrm{g} / \mathrm{ml}$ ) significantly increased the levels of the secreted MMP-9 proteins. These results suggest that AGEs are able to increase the levels of MMP-9 protein secreted into the medium.

Pioglitazone is able to reduce the high levels of MMP-9 protein induced by AGE. In order to determine whether 
A

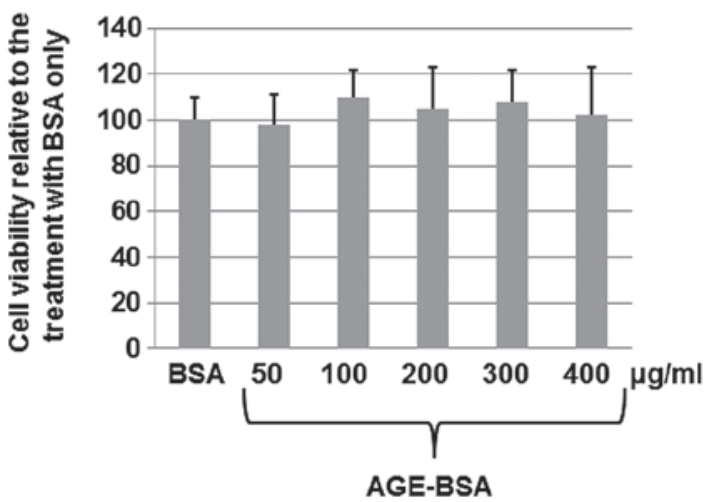

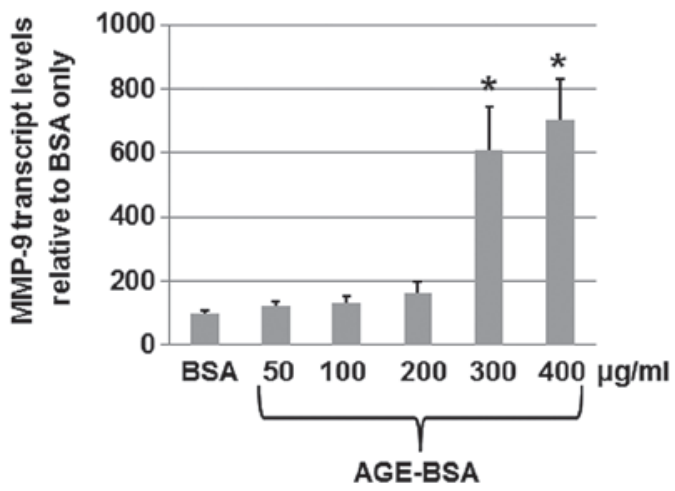

Figure 1. AGE-BSA induces the expression of MMP-9 in HaCaT cells. HaCaT cells at a density of 6x105 cells/well were seeded into 6-well plates, serum-starved overnight and then cultured for $24 \mathrm{~h}$ with various concentrations $(50,100,200,300$ and $400 \mu \mathrm{g} / \mathrm{ml}$ ) of AGE-BSA or unmodified BSA. (A) Cell viability was measured using the 3-(4,5-dimethylthiazol-2-yl)-2,5-diphenyltetrazolium bromide assay following the treatment. (B) Total RNA was harvested from HaCaT cells treated with AGE-BSA or unmodified BSA only. Quantitative polymerase chain reaction was performed to analyze the MMP-9 mRNA levels in the cells treated with various concentrations (50, 100, 200, 300 and $400 \mu \mathrm{g} / \mathrm{ml}$ ) of AGE-BSA compared with the BSA only control. The levels (mean value) of MMP-9 transcripts in cells were calculated. Error bars show the mean \pm standard deviation $(\mathrm{P}<0.05)$. The experiments were repeated at least three times. ${ }^{*} \mathrm{P}<0.05$ vs. the corresponding control. MMP-9, matrix metalloproteinase-9; AGE, advanced glycation end-products; BSA, bovine serum albumin.

A

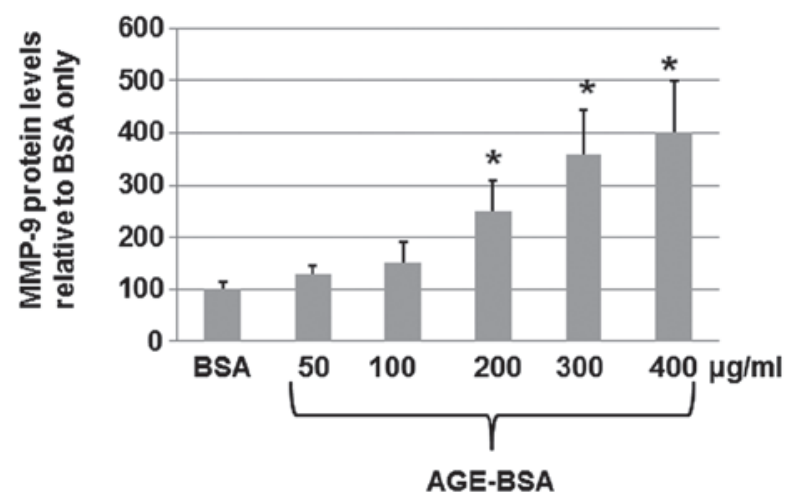

B

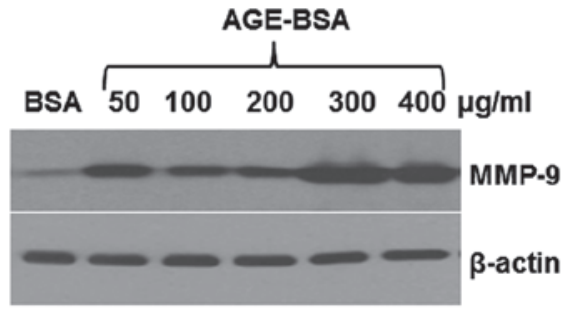

Figure 2. Levels of MMP-9 protein in the medium as determined by ELISA and western blot analysis. HaCaT cells at a density of $6 x 10^{5}$ cells/well were seeded into 6-well plates, serum-starved overnight and then cultured for $24 \mathrm{~h}$ with various concentrations (50, 100, 200, 300 and 400 $\mu \mathrm{g} / \mathrm{ml})$ of AGE-BSA or unmodified BSA. (A) Medium was collected $24 \mathrm{~h}$ post-treatment with AGE-BSA or unmodified BSA. The levels of MMP-9 in the culture were detected by ELISA. The data (mean \pm standard deviation) are from six independent experiments. (B) Medium was collected and concentrated. The total proteins were isolated from the medium and subjected to western blot analysis. Primary antibodies against MMP-9 and $\beta$-actin were purchased from Santa Cruz Biotechnology, Inc, Santa Cruz, CA, USA). The secondary antibodies used in the present study were goat anti-mouse IgG conjugated to horseradish peroxidase. Bound antibodies were detected using an enhanced chemiluminescence system (Pierce Biotechnology, Inc.). The experiments were repeated independently at least three times. Image quantifications were performed using ImageQuant software. MMP-9, matrix metalloproteinase-9; ELISA, enzyme-linked immunosorbent assay; AGEs, advanced glycation end-products; BSA, bovine serum albumin.

pioglitazone is able to decrease MMP-9 expression, HaCaT cells at a density of $6 \times 10^{5}$ cells/well were serum-starved overnight and then cultured for $24 \mathrm{~h}$ with $300 \mu \mathrm{g} / \mathrm{ml}$ AGE-BSA in the absence or presence of pioglitazone $(0.5$ or $1 \mu \mathrm{M})$. The total proteins were harvested from the cells and then subjected to western blot analysis. As shown in Fig. 3A, pioglitazone $(0.5 \mu \mathrm{M})$ significantly inhibited the MMP-9 level. A higher concentration of pioglitazone $(1 \mu \mathrm{M})$ resulted in a greater inhibitory effect on the MMP-9 level. The levels of MMP-9 in the medium were also measured by ELISA. As shown in Fig. 3B, pioglitazone $(0.5$ or $1 \mu \mathrm{M})$ significantly suppressed the levels of MMP-9 in the medium. These results suggest that pioglitazone significantly inhibits the expression of MMP-9.
Pioglitazone reduces the protein expression of MMP-9 induced by AGEs. To further investigate the mechanisms underlying the inhibitory effect of pioglitazone on the increased MMP-9 expression induced by AGEs, HaCaT cells at a density of $6 \times 10^{5}$ cells/well were serum-starved overnight and then cultured for $24 \mathrm{~h}$ with $300 \mu \mathrm{g} / \mathrm{ml}$ AGE-BSA in the absence or presence of pioglitazone $(0.5$ or $1 \mu \mathrm{M})$. Total RNA was harvested from $\mathrm{HaCaT}$ cells and qPCR was performed to analyze the mRNA levels of MMP-9. As shown in Fig. 4, the $\mathrm{qPCR}$ results indicated that pioglitazone at concentrations of 0.5 and $1 \mu \mathrm{M}$ significantly suppressed the levels of MMP-9 mRNA to 20 and $8 \%$, respectively. These results suggest that pioglitazone suppresses the expression of MMP-9 via a transcriptional mechanism. 
A

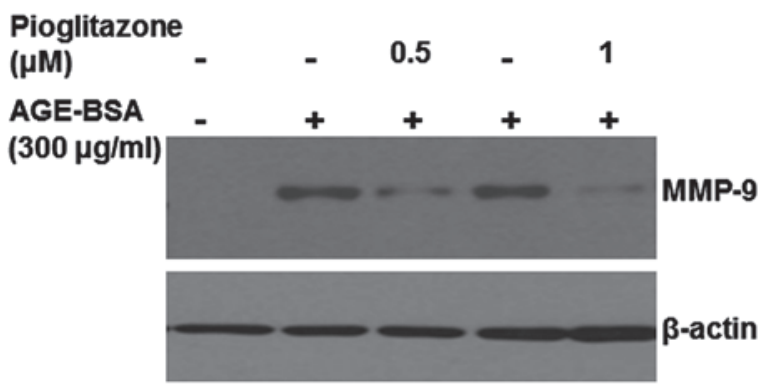

B

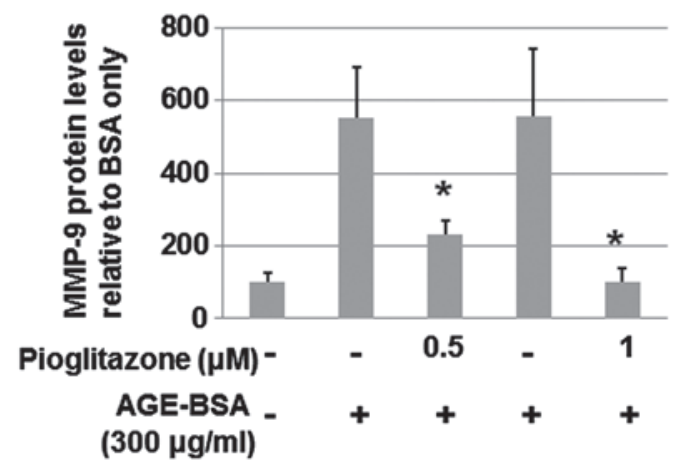

Figure 3. Effects of pioglitazone on the high level of MMP-9 protein induced by AGE. HaCaT cells at a density of $6 \times 10^{5}$ cells/well were serum-starved overnight and then cultured for $24 \mathrm{~h}$ with $300 \mu \mathrm{g} / \mathrm{ml}$ AGE-BSA in the absence or presence of pioglitazone $(0.5$ or $1 \mu \mathrm{M})$. (A) Total proteins were harvested from the cells and then subjected to western blot analysis. (B) The levels of MMP-9 in the medium were also measured by enzyme-linked immunosorbent assay. The experiments were repeated at least six times. ${ }^{*} \mathrm{P}<0.05$, compared with the cells treated with AGE-BSA. MMP-9, matrix metalloproteinase-9; AGEs, advanced glycation end-products; BSA, bovine serum albumin.

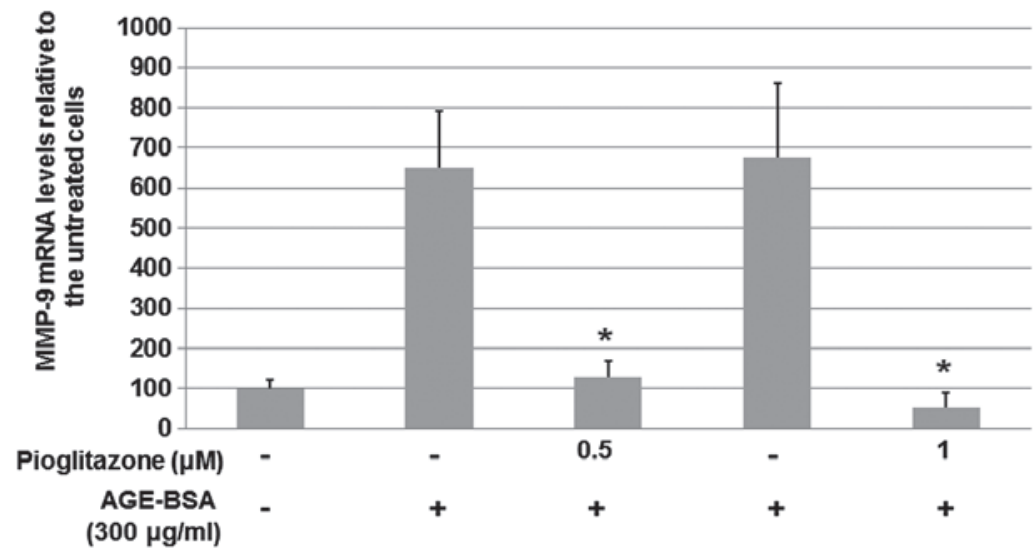

Figure 4. qPCR detection of MMP-9 mRNA. HaCaT cells at a density of $6 \times 10^{5}$ cells/well were serum-starved overnight and then cultured for 24 h with $300 \mu \mathrm{g} / \mathrm{ml}$ AGE-BSA in the absence or presence of pioglitazone $(0.5$ or $1 \mu \mathrm{M})$. Total RNAs were harvested from HaCaT cells. qPCR was performed to analyze the MMP-9 mRNA levels in the cells. The levels (mean value) of MMP-9 transcripts in the cells were calculated. Error bars show the mean \pm standard deviation $(\mathrm{P}<0.05)$. The experiments were repeated at least three times. ${ }^{*} \mathrm{P}<0.05$, when compared with the cells treated with AGE-BSA. qPCR, quantitative polymerase chain reaction; MMP-9, matrix metalloproteinase-9; AGEs, advanced glycation end-products; BSA, bovine serum albumin.

\section{Discussion}

As a PPAR agonist, pioglitazone has demonstrated promise as a therapeutic agent due to its ability to increase the functional recovery of wounds and decrease lesion sizes following injury (21). The therapeutic effects of pioglitazone are considered to be a result of the regulation of multiple pathways (11-16). Since the increased expression of MMP-9 contributes to poor wound healing in diabetic foot ulcers $(4,5)$, the present study examined whether pioglitazone acts via a mechanism associated with the regulation of the expression of MMP-9 in human keratinocytes treated with AGEs. The results revealed that pioglitazone at concentrations of 0.5 or $1 \mu \mathrm{M}$ suppressed the levels of MMP-9 mRNA to 20 or 8\%, respectively. Since the increased expression of MMP-9 contributes to poor wound healing $(4,5)$, the results of the present study suggest that pioglitazone may have therapeutic effects on impaired wound healing associated with diabetes via a mechanism of inhibiting MMP-9 expression. This finding provides novel evidence for the application of pioglitazone in the field of wound healing.
It is reported that pioglitazone may inhibit the TGF- $\beta$-induced myofibroblast differentiation (22). Pioglitazone also attenuates TGF- $\beta$-induced type I collagen and fibronectin mRNA and protein production, which are involved in burn wound healing $(22,23)$. It was revealed that PPAR- $\gamma$-dependent and PPAR- $\gamma$-independent mechanisms were involved in the action of pioglitazone (22-24), although PPAR- $\gamma$ agonists do not prevent the activation of quiescent hepatic stellate cells in vitro, nor hepatic fibrogenesis in mice (24).

In addition to the diabetes-associated wound healing, pioglitazone may be useful for other types of wound healing, including gastric ulcer healing. The involvement of PPAR- $\gamma$ in inflammatory responses during pioglitazone-mediated gastric ulcer healing has been reported (25). In the present study, the findings suggest that use of pioglitazone has potential in the wound healing therapy and it may be a promising approach upon further study. The results provide novel evidence for understanding the molecular mechanisms underlying the action of pioglitazone. 


\section{Acknowledgements}

This study was supported by the Inner Mongolia Institute of Burn.

\section{References}

1. Gill SE and Parks WC: Metalloproteinases and their inhibitors: regulators of wound healing. Int J Biochem Cell Biol 40: 1334-1347, 2008

2. Yang C, Zhu P, Yan L, et al: Dynamic changes in matrix metalloproteinase 9 and tissue inhibitor of metalloproteinase 1 levels during wound healing in diabetic rats. J Am Podiatr Med Assoc 99: 489-496, 2009.

3. Reiss MJ, Han YP, Garcia E, et al: Matrix metalloproteinase-9 delays wound healing in a murine wound model. Surgery 147: 295-302, 2010

4. Liu Y, Min D, Bolton T, et al: Increased matrix metalloproteinase-9 predicts poor wound healing in diabetic foot ulcers. Diabetes Care 32: 117-119, 2009.

5. Rayment EA, Upton Z and Shooter GK: Increased matrix metalloproteinase-9 (MMP-9) activity observed in chronic wound fluid is related to the clinical severity of the ulcer. Br J Dermatol 158 951-961, 2008

6. Bell DS: Beta-cell rejuvenation with thiazolidinediones. Am J Med 115: 20S-23S, 2003

7. Saitoh Y, Chun-ping C, Noma K, Ueno H, Mizuta M and Nakazato M: Pioglitazone attenuates fatty acid-induced oxidative stress and apoptosis in pancreatic beta cells. Diabetes Obes Metab 10: 564-573, 2008.

8. Liu X, Luo D, Zheng M, Hao Y, Hou L and Zhang S: Effect of pioglitazone on insulin resistance in fructose-drinking rats correlates with AGEs/RAGE inhibition and block of NADPH oxidase and NF kappa B activation. Eur J Pharmacol 629: $153-158,2010$

9. Ao C, Huo Y, Qi L, Xiong Z, Xue L and Qi Y: Pioglitazone suppresses the lipopolysaccharide-induced production of inflammatory factors in mouse macrophages by inactivating NF-kappaB. Cell Biol Int 34: 723-730, 2010.

10. Wan H, Yuan Y, Qian A, Sun Y and Qiao M: Pioglitazone, a PPARgamma ligand, suppresses NFkappaB activation through inhibition of IkappaB kinase activation in cerulein-treated AR42J cells. Biomed Pharmacother 62: 466-472, 2008.

11. Bell DS: Beneficial effects resulting from thiazolidinediones for treatment of type 2 diabetes mellitus. Postgrad Med 8: 35-44, 2003 .
12. Gumieniczek A, Hopkała $\mathrm{H}$ and Zabek A: Protective effects of a PPARgamma agonist pioglitazone on anti-oxidative system in testis of diabetic rabbits. Pharmazie 63: 377-378, 2008.

13. Collino M, Aragno M, Mastrocola R, et al: Modulation of the oxidative stress and inflammatory response by PPAR-gamma agonists in the hippocampus of rats exposed to cerebral ischemia/reperfusion. Eur J Pharmacol 530: 70-80, 2006.

14. Gumieniczek A, Krzywdzińska M and Nowak M: Modulation of nitrosative/oxidative stress in the lung of hyperglycemic rabbits by two antidiabetics, pioglitazone and repaglinide. Exp Lung Res 35: 371-379, 2009.

15. Somi MH, Hajipour B, Asl NA, et al: Pioglitazone attenuates ischemia/reperfusion-induced liver injury in rats. Transplant Proc 41: 4105-4109, 2009.

16. Bernardo A, Bianchi D, Magnaghi V and Minghetti L: Peroxisome proliferator-activated receptor-gamma agonists promote differentiation and antioxidant defenses of oligodendrocyte progenitor cells. J Neuropathol Exp Neurol 68: 797-808, 2009.

17. Fukami K, Yamagishi SI, Sakai K, Kaida Y, Adachi T, Ando R and Okuda S: Potential inhibitory effects of L-carnitine supplementation on tissue advanced glycation end products in patients with hemodialysis. Rejuvenation Res 16: 460-466, 2013.

18. Goldin A, Beckman JA, Schmidt AM and Creager MA: Advanced glycation end products: sparking the development of diabetic vascular injury. Circulation 114: 597-605, 2006.

19. Bierhaus A and Nawroth PP: Multiple levels of regulation determine the role of the receptor for AGE (RAGE) as common soil in inflammation, immune responses and diabetes mellitus and its complications. Diabetologia 52: 2251-2263, 2009.

20. Wautier JL and Guillausseau PJ: Advanced glycation end products, their receptors and diabetic angiopathy. Diabetes Metab 27: 535-542, 2001

21. Yonutas HM and Sullivan PG: Targeting PPAR isoforms following CNS injury. Curr Drug Targets 14: 733-742, 2013.

22. Pan HW, Xu JT and Chen JS: Pioglitazone inhibits TGF $\beta$ induced keratocyte transformation to myofibroblast and extracellular matrix production. Mol Biol Rep 38: 4501-4508, 2011.

23. Dong X, Geng Z, Zhao Y, Chen J and Cen Y: Involvement of mast cell chymase in burn wound healing in hamsters. Exp Ther Med 5: 643-647, 2013

24. Da Silva Morais A, Abarca-Quinones J, Horsmans Y, Stärkel P and Leclercq IA: Peroxisome proliferated-activated receptor gamma ligand, Pioglitazone, does not prevent hepatic fibrosis in mice. Int J Mol Med 19: 105-112, 2007.

25. Lahiri S, Sen T and Palit G: Involvement of glucocorticoid receptor and peroxisome proliferator activated receptor-gamma in pioglitazone mediated chronic gastric ulcer healing in rats. Eur J Pharmacol 609: 118-125, 2009. 